\title{
Assessing re-establishment of functional forest ecosystems on reclaimed oil sands mine lands
}

\author{
J. Straker Integral Ecology Group Ltd., Canada; and Reclamation Working Group, Cumulative \\ Environmental Management Association, Canada \\ G. Donald Donald Functional \& Applied Ecology Inc.; and Reclamation Working Group, Cumulative \\ Environmental Management Association, Canada
}

\begin{abstract}
Oil sands mining in Northeastern Alberta occurs on a predominantly forested boreal landscape, across tens of thousands of hectares. One of the fundamental end goals of oil sands mine reclamation and closure is the re-establishment on this landscape of functional forested ecosystems, and the end land uses that these ecosystems support (i.e., commercial forestry, traditional use, wildlife habitat, recreation). Such reestablishment relies on the generation and refinement of knowledge on the requisite factors for successful forest development on reclaimed lands, and on the ability to make informed projections of future forest characteristics based on current conditions. Some of this knowledge development occurs through the Cumulative Environmental Management Association's Reclamation Working Group (RWG), a multistakeholder organisation with members from government, industry, regulatory bodies, environmental groups and Aboriginal groups, which is tasked with developing frameworks and guidance documents containing recommendations regarding mine reclamation practices in the oil sands region.
\end{abstract}

This paper presents information on RWG's approach to the guidance and assessment of reforestation of oil sands mine disturbances, with particular attention to four programmes under development which form components of this approach, as follows:

- Information contained in Guidelines for Reclamation to Forest Vegetation in the Athabasca Oil Sands Region, 2nd ed., on species selection and establishment techniques/density for forest vegetation.

- Assessment of forest stand juvenile establishment and growth performance in the Alternative Regeneration Standards for reclaimed oil sands mine areas.

- Work completed by RWG to establish measures of forest stand productivity for reclaimed sites, based on similar metrics in surrounding stands initiated through forest harvest or wildfire.

- An initiative to determine methods for modelling forest stand development on reclaimed oil sands mine sites.

This paper will also discuss some evolving conceptual aspects of oil sands mine reforestation and assessment, including examination of:

- Expectations for establishment of productive forests capable of meeting commercial utilisation standards immediately following mining.

- Whether the industrial forestry assessment paradigm is appropriate for application to reclaimed oil sands mines, or whether this unique situation demands an equally unique approach to stand regeneration and evaluation.

\section{Introduction}

The Athabasca Oil Sands Region (AOSR) is currently estimated to contain the world's second-largest recoverable crude-oil deposits, and is located in the northeast of the province of Alberta, in an area of largely intact and ecologically important boreal forest with a small human population. The majority of oil sands extraction to date in this region has occurred through surface mining, which creates substantial land 
disturbance requiring land reclamation, defined as "the stabilisation, contouring, maintenance, conditioning, reconstruction, and revegetation of the surface of the land to a state that permanently returns the plant to a land capability equivalent to its pre-disturbed state," (RSA 2000, c.E-12, approval no. 151469-00-01, Section 1.1.2 (11)). The current surface mining footprint is approximately 70,000 hectares (ha), and projections of future extraction activity indicate that the eventual disturbance from surface mining of oil sands in the AOSR could reach 200,000-300,000 ha. To date, approximately 7,000 ha are documented as reclaimed (Government of Alberta, 2011).

As a result of increasing industrial activity in the AOSR, the Cumulative Environmental Management Association (CEMA) was formed in 2000. CEMA is a multi-stakeholder organisation with members from government regulatory agencies, mining and other industrial operators, and non-governmental organisations, including local/indigenous communities. CEMA is tasked with developing frameworks to manage the cumulative environmental effects of regional development on air, land, water and biodiversity.

One of CEMA's committees is the Reclamation Working Group (RWG), which produces and maintains guidance documents that provide recommendations on regional mine reclamation practices. These guidance documents are mandated by industry operational regulatory approvals, and steward towards the overall goal that reclaimed landscapes meet regulatory requirements, satisfy the needs and values of stakeholders, and are environmentally sustainable. One of RWG's tasks is to provide guidance on the re-establishment on reclaimed landscapes of forest ecosystems, which make up the majority of the pre-disturbance landscape, and support a variety of human land uses, including commercial forestry, traditional land use by regional aboriginal populations, and recreation.

\section{Background - Reclamation Working Group}

There are two aspects of RWG's operation that are foundational to the successful development of its products, including work and recommendations on forest reclamation:

1. Multi-stakeholder participation, with consensus-based decision making - RWG and its subgroups are populated by representatives from participating member organisations, as well as by a limited number of CEMA staff. Representatives (and some staff members) are technical specialists who are charged with the duties to both actively contribute to the development, implementation, and completion of the technical work programme; and to represent their organisations' interests during this process. Completion of the RWG work programme is achieved through a variety of mechanisms, including out-sourced contracts, technical work by CEMA staff, and technical work and writing by member representatives. This active involvement and contribution by members leads to significant ownership of and commitment to Working Group products. Reinforcing this commitment is that fact that all CEMA recommendations to the Alberta Government (e.g., new guidance manuals) must have consensus approval at multiple levels in the recommendation process. This consensus-based decision structure is at times unwieldy, but ultimately contributes to improved understanding of various member-organisations' motivations and constraints, and can produce guidance documents that are informed and shaped by a variety of interests.

2. Support in the form of technical information from a broad network of research and monitoring programmes - RWG's products are supported by results from a wide variety of initiatives. RWG itself conducts work targeted to address specific issues of uncertainty in reclamation (e.g., research into the development of eco-hydrological function on reclaimed sites, and estimates of early stand mortality on reclaimed forest lands), as well as maintaining a long-term network of monitoring plots on reclaimed sites and in non-mined reference ecosystems. In addition, RWG has access to information generated through individual and collaborative industry reclamation-research programmes across the AOSR (note that the majority of this work, both CEMA and external, is funded by the oil sands industry). Information from all applicable programmes is discussed and synthesised at RWG, and used to inform development of Working Group products. Thus most RWG deliverables are supported by 10 or more years of reclamation research and monitoring programmes investigating the establishment and development of forest vegetation communities on reclaimed oil sands sites, and on the materials and processes that influence this establishment and development. 


\section{Objectives}

This paper has two primary purposes:

1. To present work that is recently completed or ongoing within RWG that is designed to advance knowledge of forest reclamation in the AOSR, and thus to improve reclamation guidance and practice in the region.

2. To discuss the broader context of forest reclamation, including an exploration of our application of reforestation approaches from the forestry industry to the mine reclamation setting, our concepts of post-closure "equivalent land capability" to support "end land uses", and our desire to maintain the capacity for extractive activities on all landscapes at all times.

\section{$4 \quad$ RWG forest reclamation initiatives}

\subsection{Guidelines for reclamation to forest vegetation in the Athabasca Oil Sands Region}

In 1998, RWG's multi-stakeholder predecessor (the Oil Sands Vegetation Reclamation Committee) produced the first version of the Guidelines for Reclamation to Forest Vegetation in the Athabasca Oil Sands Region (Oil Sands Vegetation Reclamation Committee, 1998), locally referred to as the "Revegetation Manual". The focus of this manual was to provide guidance on "starter vegetation and design criteria for ecosystems that would support primarily commercial forests [emphasis added] and secondarily would provide wildlife habitat". This document stated that it was based on "successful reclamation techniques" used at the time of document preparation, and would be updated as new data from research and monitoring programmes became available to refine techniques. This first version of the Revegetation Manual provided estimates of reclaimed soil moisture and nutrient regimes, based on current mine-waste disposal and coverplacement practices and on landform/topographic position. Estimates of soil moisture and nutrient regimes were then used to provide guidance on overstory and understory species selection and planting densities, again based on the practices utilised at the time at the two operational mines in the region.

In 2010, Alberta Environment published the second edition of the Revegetation Manual, which was revised and developed by the Reclamation Working Group. The introduction of this 2nd edition states:

- The goal of the Revegetation Manual is to provide guidance on re-establishing the vegetation component of upland ecosystems on reclaimed landscapes, and on evaluating the success of this reestablishment. This goal is based on the following fundamental concepts:

- That reclaimed plant communities should have species characteristic of native plant communities in the oil sands region.

- That trends of vegetation community and structure development on reclaimed landscapes should be similar to native plant communities in the oil sands region.

- That reclaimed ecosystems should have developmental trajectories that satisfy land-use objectives, and have characteristics that provide resilience against natural disturbance events (Alberta Environment, 2010).

Differences between the 1st and 2nd editions of the Revegetation Manual are partially attributable to advances in reclamation knowledge developed over the 12-year period between their publication dates, but also show some changes in overall approach, as reflected in their respective goal/focus statements. These differences in approach are due to a host of shifting factors in the oil sands region, including evolving stakeholder (industrial, regulatory, community, and non-governmental organisations) expectations, and related increased provincial, national, and international attention on oil sands development and reclamation. More important differences between the 1st and 2nd editions of the Revegetation Manual include:

- More rigorous and/or explicit methods used in the 2 nd edition to derive estimates of soil moisture and nutrient regimes, based on a companion document, the Land Capability Classification System for Forest Ecosystems in the Oil Sands, 3rd ed. (CEMA, 2006). 
- Inclusion in the 2 nd edition of wider range of appropriate vegetation understory species for forest reclamation, based on an increased emphasis on re-establishment through reclamation of native vegetation communities, and on acknowledgement of wider amplitudes of ecological occurrence for individual species than those employed in the first edition. This inclusion is supported by accompanying information on ecology and propagation and establishment techniques for a number of listed understory species.

- Inclusion in the 2nd edition of a wider range of overstory (tree) establishment densities, based on recognition that reclamation practitioners may want to establish a variety of forest conditions through their actions (e.g., sparse stands for understory biodiversity, which may support different wildlife habitats and increased potential for aboriginal traditional use; or more densely stocked stands, for more rapid litter deposition and soil development, and/or forest fibre production values). These ranges were developed based on a transparent forest stand-development and growth modelling approach (Timberline Natural Resource Group Ltd., 2009), rather than documentation of current practices, as was used in the 1 st edition.

- Inclusion in the 2nd edition of guidance on salvage and use of forest-floor and surface-soil materials, with the objective of preserving and replacing vegetation propagules in these materials, and thus increasing natural regeneration of native vegetation species on reclaimed sites.

- Inclusion in the 2nd edition of guidance on assessment of revegetation success, based on a range of indicators incorporating measures of native species presence and change over time, net primary production, and overstory characteristics (as assessed through regeneration standards, discussed below).

With its information and guidance on selection, establishment, and assessment of vegetation overstory and understory species for forested ecosystems, the Revegetation Manual 2nd edition is a primary component of the current reclamation approach for oil sands mine disturbances in the AOSR.

\subsection{Ecosite area summary table}

Historic regulatory operating approvals for oils sands mines in the AOSR (and most, if not all, current approvals) include conditions that operators provide information on expected timber productivity on reclaimed landscapes, in the format of area (in ha) per "Timber Productivity Rating" ("TPR") class. This rating system was generally based on assigning reclaimed areas to forest growth-and-yield classes (measured by expected "site index", or projected height of the largest 100 trees per ha at a breast-height age of 50 years), and then providing expected total ha per class. Following issuance of a number of operating approvals in 2007, RWG members began discussing the applicability of the TPR concept to evolving ideas of reclaimed forest productivity, and preferable methods of reflecting forest reclamation goals in operating approvals. To this end, the group began developing an "ecosite area summary table" as a replacement for TPR tables in operating approvals. (An ecosite is a unit of ecological classification in Alberta, which can be defined as a functional classification unit based on the combined interaction of biophysical factors which together dictate the availability of moisture and nutrients for plant establishment and growth.)

The rationale for the shift away from a TPR- to an ecosite-based approach is founded on the contrast between the two concepts:

- TPR is seen to be largely uni-dimensional, simply describing expected growth of dominant trees in a stand over a relatively short time period.

- Ecosite is a more holistic or multi-variate unit, describing expected edaphic conditions and corresponding vegetation species presence and other responses to these conditions, including the growth-over-time aspect captured by the TPR concept.

Ecosites also form the planning basis of the Revegetation Manual and of vegetation closure-planning processes used by mine operators. Thus, RWG's intention was that a shift to an ecosite-based table to describe expected reclaimed forest conditions in operating approvals would both: 
- Rationalise a number of parallel or overlapping planning exercises (e.g., closure planning, use of guidance presented in the 2nd edition of the Revegetation Manual, approval development, and implementation of reclamation plans) through the use of the same ecological unit.

- Broaden the applicability beyond the TPR approach, such that information on expected plant communities would be included alongside more conventional information on expected forest growth-and-yield.

The new table proposed by RWG includes, for each ecosite in the forested regional landscape, information on leading overstory species, mean site index by species (derived from a robust examination of empirical data from non-mined forest stands in the region), and the minimum site index by species required for reclaimed stands to be considered of merchantable productivity (TECO Natural Resource Group Limited, 2011a). The intended use of this table is that operators will populate it, for each mining operation, with area (ha) expected to be reclaimed to each ecosite (from xeric jack pine-lichen communities to subhygric white and black spruce communities), and the proportion of that area expected to meet merchantability requirements. It is also expected that as monitoring results allow, an additional column will be added for observed site index by species, which will provide an opportunity to evaluate whether growth patterns observed in pyrogenic or post-harvest stands in the region are replicated by stands on reclaimed mine lands, or whether different growth patterns are emerging on these reclaimed sites. Thus the new ecosite area summary table retains some of the commercial forest emphasis of previous operating tables, but expands the information considered and included, as each ecosite represents not only overstory productive potential, but also a range of associated understory species that would be expected to co-occur in non-mined stands in the region.

\subsection{Alternative regeneration standards}

A common approach in Canadian land reclamation that involves reforestation is the application of provincial standards developed for use in the forestry industry, based on the following premises:

1. If pre-disturbance landscapes were forested, then reclaimed landscapes should also be forested, both for ecological and ideological (i.e., replace what one disturbs) reasons, unless alternative end land uses are proposed.

2. If pre-disturbance forested landscapes were capable of supporting forests with commercial value, then reclaimed forested landscapes should also be capable of supporting forests with commercial value, again for both ecological and ideological reasons (i.e., we should be designing and implementing reclamation such that similar ecosystem functions, such as forest establishment and growth, are restored, but also have an expectation of uninterrupted capacity for extractive activities on our landscapes).

3. Reclaimed forested landscapes with potential commercial value should be capable of meeting the same standards as post-harvest lands in the non-mined forest land base.

Examples of such standards are the minimum stocking and height values described until recently in Alberta's Regeneration Survey Manual (Alberta Environmental Protection, 1994), which were designed to ensure that forest licensees had re-established after harvest forest vegetation with suitable species, densities and growth to mature into merchantable stands. Although these standards were developed solely for application to the forestry industry, it has been assumed that they should be applied to reforested reclamation sites as well, and both the 1st and 2nd editions of the Revegetation Manuals contain references to adherence to provincial regeneration standards for monitoring and evaluation of reclaimed forest land.

Over the last several years, Alberta Sustainable Resource Development (ASRD) has worked in consultation with forestry companies to develop new Provincial regeneration standards, the Regeneration Standards of Alberta (Provincial RSA). These standards were developed with the intent to create a linkage between juvenile stand conditions and future yields, where the linkage between these two states would be derived using growth model projections. Final "success" of regeneration is evaluated using projected productivity of the stand (culmination mean annual increment of merchantable timber), where projected productivity is determined using regeneration survey data as inputs to growth models. As such, the development of data 
collection protocols were primarily driven by the need to collect data suitable for model inputs, and the majority of compilation and assessment procedures are linked to model needs.

Although, as illustrated above, there has been an operative premise that provincial regeneration standards developed for use in the forestry industry should be applied to reclaimed forested mine lands, there is a growing recognition that these standards are not necessarily directly suitable for this application, for two primary reasons:

1. Differences in knowledge - the provincial regeneration standards rely on the use of forest growthand-yield models, which have a relatively robust empirical basis for application on pyrogenic and post-harvest stands, but which have not been tested for applicability to reclamation areas.

2. Differences in scope - although forest licensees may manage large land bases which include nonmerchantable forest, they harvest merchantable stands, and thus application of regeneration standards on post-harvest areas is restricted to areas that are expected to be merchantable. In contrast, forest reclamation of oil sands mines may occur for a variety of reasons or objectives (e.g., re-establishing certain forest plant communities), which may or may not include re-establishment of merchantable forest stands. Thus, application of a relatively narrow assessment model based on regeneration of commercial forests to a landscape that is expected to meet a suite of broader objectives may not be the ideal approach.

In order to develop an approach more directly designed for oil sands reclamation, the Alberta government agreed that RWG should undertake work to develop regeneration standards specific to forest reclamation of mine land in the AOSR. The initial component of this work, developing reclamation-specific sampling protocols, has been accomplished by modifying the Provincial RSA to meet needs for assessment of reclaimed lands (TECO Natural Resource Group Limited, 2011b). Primary differences in the reclamation sampling protocols (in comparison to survey methods used by forest licensees) include extending the survey window to 20 years following planning (instead of the typical 14-year window), in recognition that reclamation forest stands may develop more slowly than their post-harvest counterparts, or may take longer to reach an equilibrium state in which survey would provide representative information; and modification of sampling-intensity guidelines for smaller sampling units, as reclamation tends to produce a larger number of smaller reforested polygons than forest harvest. In addition, although these reclamation-specific protocols are still primarily focused on testing against criteria for eventual merchantability, they have been developed to more broadly yield useful information to evaluate the successful establishment of forest stands, whether merchantable or not, on the reclaimed landscape. The developed protocols were field-tested in the summer of 2010, and modified based on this testing. The next anticipated step in developing this product is to confirm the corresponding standards or threshold values against which survey data will be assessed.

\subsection{Modelling forest stand development}

As noted in the above discussion, one of the primary limitations to understanding development of forests on reclaimed lands, and to confident projection of likely future characteristics of these forests, is the lack of precedent in this type of reforestation, and a corresponding scarcity of observed data on which to base projections. In Canada's boreal forest, we possess relatively robust datasets on forest stand development following pyrogenic disturbance, and are assembling comparable datasets on post-harvest stand development, but lack observations on long-term forest development following mining, which requires largescale reconstruction of landforms and soils in addition to revegetation. In the absence of such data, our approach has been to apply forest-development models based on other (less severe) disturbances to reclaimed mine land, but do not know whether this application will prove valid over the long-term (i.e., the timeframe from forest initiation to maturity), and have limited data to test the validity of this application. In fact, we know that there are likely important differences between reclaimed and non-mined forest stands: reclaimed sites occur on engineered structures using reconstructed soils and mine waste materials, rather than on "natural" or non-anthropogenic landscapes, and thus have different initial characteristics - including soil hydrology, soil chemistry and soil structure - than these natural landscapes. These differences and resulting uncertainties are compounded by the fact that reclaimed areas are in the juvenile stages of landform and soil development, and the characteristics listed above have been demonstrated to change rapidly in these stages. Thus, natural observable forest analogues are not directly comparable to reclaimed forests. The 
characteristics we directly observe on these reclaimed sites may not be in equilibrium, and can be expected to change, sometimes substantially, over the intended period of our projections. In addition, some of our management practices, particularly chemical fertilisation, may impact reclaimed-site productivity, meaning that observed early forest stand development on these sites is not necessarily indicative of long-term productivity.

In response to this, RWG has initiated a project to explore the advancement of forest-development forecast modelling specific to oil sands mine reclamation. The principal challenges of this endeavour are recognised from the outset, i.e., empirical models, which are largely constructed on long-term observations, will struggle with the lack of long-term data, while process-based models will struggle with the extrapolation of processes observed in non-mined forests to reclaimed systems where those processes are known to be different.

Initial objectives of RWG's forest-development modelling project are to review existing models and approaches, to gain an understanding of the opportunity for application and modification of these models to reclaimed forest ecosystems. Specifically, RWG is evaluating, in a reclamation context, the potential of existing models to:

1. Project:

a. Single-species yields (commercial tree vegetation).

b. Forest-stand development (including non-dominant tree species, shrubs, grasses, forbs) over time.

2. Use observed (juvenile) tree growth to assess and project longer-term forest productivity.

3. Provide information on vegetation community development, including the relationships between canopy trees and understory shrubs, and how those relationships change or influence forest-stand development.

4. Address identified parameters thought to be important in determining reclaimed vegetation responses (e.g., topography; slope gradient, position and aspect; solar radiation; soil depth; soil temperature; soil moisture; salinity; etc.).

RWG intends that this effort will improve our ability to understand ranges of probable future forest conditions on reclaimed landscapes and the factors that influence these conditions, and will expand our conceptualisation of forest development from one focused on fibre growth-and-yield to one more inclusive of the range of vegetation species that comprise boreal forests.

\subsection{Stakeholder acceptance of these approaches}

An advantage of RWG's structure is that interested stakeholders, including regulatory authorities, have the opportunity to be directly involved in development of RWG recommendations to government. Thus, all of the above examples of RWG initiatives, which are of direct relevance to forest land management in northeast Alberta, have involved extensive participation and input from Alberta Sustainable Resource Development, which is the agency that will ultimately oversee their implementation. It is RWG's experience that this inclusive process helps to ensure understanding and acceptance of RWG products, and effective incorporation of these products into Alberta's regulatory regime.

\section{Conceptual aspects of oil sands mine reforestation and assessment}

The RWG projects described in Section 4 contribute to the generation and refinement of knowledge for successful forest development on reclaimed lands, and on the ability to make informed projections of future forest characteristics based on current conditions. The broader context of forest reclamation, including an exploration of our application of reforestation approaches from the forestry industry to the mine reclamation setting, our concepts of post-closure "equivalent land capability" to support "end land uses", and our desire to maintain the capacity for extractive activities on all landscapes at all times are considered with respect to the four projects described previously. 


\subsection{Application of reforestation approaches from the forestry industry to the mine reclamation setting}

As discussed above, a fundamental approach to evaluating forest reclamation in northeast Alberta has been to use standards and assessment methodologies developed for the forestry industry, on the assumption that reclaimed forest ecosystems will replicate functions, forms and processes found in non-mined forests in the region. In the reclamation context, there are two limitations to this approach:

1. We do not know if it is correct - given the significant difference in the severity and contiguous scale of disturbance of oil sands mining in comparison to forest harvest, the assumptions and empirical relationships defined in forestry-industry standards and assessment methods need to be tested on reclaimed oil sands mine lands to confirm their applicability. Predictions of the development, sustainability and resilience of reclamation areas are based on non-mined forest empirical relationships or process-based modelling approaches which have not been validated for oil sands mine reclamation. As discussed above, there are a number of important differences between reclaimed landscapes and non-mined forest landscapes, and these differences make it difficult to know at this time whether assumptions of analogy to more familiar forest development patterns are accurate, and if forest reclamation areas will develop similarly to pyrogenic or post-harvest areas.

2. We do not know how useful it is - the guidance in the 2nd edition of the Revegetation Manual and the context behind the ecosite area summary table is grounded in the concept that, rather than focussing primarily on a goal of replacement of commercial forest, the principal reforestation objective in oil sands reclamation should be re-establishment of native forest vegetation communities and long-term ecosystem productivity (where this productivity can be defined as the capacity of a reclaimed site to support forest ecosystems over generations of humans and trees, as measured against some defined reference). The shift in focus highlights an advancement in reclamation practices, using ecosystem-, instead of land-use-, based approaches. So, given that we are a) no longer focussing on forest-stand merchantability as the primary indicator of forest reclamation success; and b) not certain whether the assumption of analogy to pyrogenic or postharvest stands is truly an apt one, we need to develop a range of planning and assessment tools broader than those conventionally used, which were targeted to re-establishment of fully stocked stands and projected timber productivity ratings. An assumption of portability of tools from the forest industry may not fully serve the need of developing a broader set of reclamation-focussed tools.

It is likely that many of the techniques needed for reclamation planning and assessment will continue to be adapted from the forestry industry, as this sector works to meet new management objectives such as maintenance of biodiversity. As such, there will be an ongoing need to evaluate long-term fidelity or deviation of reclaimed forest development to conceptual/numeric models from non-mined forests. In addition, there will be a need for new, reclamation-specific tools to address unique aspects of these anthropogenic landscapes.

As a component of developing a broader range of ecosystem-based approaches to reclamation planning and assessment, RWG has recognised that protocols and methodologies to measure diversity, vegetation structure and ecological processes in reclaimed areas are required, and that, as above, these attributes need to be assessed against defined reference conditions. In other work, not discussed in this paper, RWG is developing a criteria-and-indicators framework for reclamation certification and a supporting monitoring approach to define reference conditions and appropriate procedures for comparing reclaimed sites to reference sites. Further, in order to broaden the evaluation of reclamation success, RWG has acknowledged that developed assessment procedures need to be expanded to focus on attributes that provide information on the establishment of functional forest ecosystems supporting multiple end land uses, often simultaneously.

\subsection{Concepts of post-closure "Equivalent Land Capability" to support "End Land Uses"}

The oil sands mining reclamation regulatory context is outlined in the Environmental Protection and Enhancement Act (EPEA; RSA 2000 C12, Part 6), EPEA operating approvals (see RSA 2000, c.E-12, approval no. 151469-00-01, Part 6, for example) and the conservation and reclamation regulation (AR 115/1993). Approval conditions related to the projects described above are highlighted as examples to 
provide the conceptual aspects regarding the regulatory context, the reclamation planning and assessment context, and to facilitate an examination of the appropriateness of this approach to re-establishing functional forest ecosystems on reclaimed oil sands mine lands.

The EPEA approvals state the operators 'shall reclaim the land so that the reclaimed soils and landforms are capable of supporting a self-sustaining, locally common boreal forest regardless of end land use' - this phrase has been adopted by RWG as the goal of reclamation in the criteria-and-indicators framework project currently under development. The EPEA conservation and reclamation regulation states that "the objective [emphasis added] of conservation and reclamation of specified land is to return the specified land to an equivalent land capability." Equivalent land capability is defined as: "the ability of the land to support various land uses after conservation and reclamation is similar to the ability that existed prior to an activity being conducted on the land, but that the individual land uses will not necessarily be identical." The Mine Reclamation Plan, a requirement of the approval, shall "detail the procedures that will be used to ensure reclamation to an equivalent land capability." The Mine Reclamation Plan must address several components such as: vegetation; land uses; forest resource considering progressive establishment of upland vegetation communities, and timber productivity ratings consistent with pre-disturbance upland communities; and fish and wildlife habitat as defined by validated habitat modelling (or other habitat assessment tools) for key species consistent with pre-disturbance capabilities; and several other components. The Life of Mine Closure Plan, another approval requirement, shall "describe the utility of the landscape and its ability to meet the various end land use goals and objectives; and provide designs for individual landforms and for the lease landscape that will target specific end land uses."

The reclamation outcomes on the post-disturbance landscape, therefore, must achieve both regulatory requirements directly addressing land-use capability and future end lands, and a suite of additional objectives captured in other approval requirements and in the 2nd edition of the Revegetation Manual to re-establish diversity, vegetation structure and ecological processes (i.e., hydrologic cycle, carbon cycle, nutrient cycles) and lead to the re-establishment of functional forest ecosystems that will provide the basis for "equivalent land capability." There is a potential tension between the ecosystem function-based approaches under development in RWG's newer projects (e.g., the Revegetation Manual and Ecosite Area Summary Table), and the older paradigm of reclamation to end land uses. Interestingly, end land uses largely describe the capacity to support human uses of the land (e.g., commercial forest harvest, aboriginal traditional use, recreation; although the end land use of wildlife habitat is an exception), while the re-establishment of functions necessary to support these uses is almost exclusively "non-human". We suggest, although this has not been a common approach, that in fact the objectives of restoration of ecosystem function and replacement of equivalent land capability for a range of end land uses is sequential and hierarchical. The "use-capability" of reclaimed lands is directly dependent on re-establishment not only of certain ecosystem characteristics, but of functional processes in these ecosystems, and human land uses are not possible or appropriate until these processes have been well established. Thus, we suggest that on reclaimed sites, there will need to be a period of re-establishment of ecosystem function prior to consideration of all but the least invasive human land uses.

\subsection{Desire to maintain the capacity for extractive activities on all landscapes at all times}

A major trade-off exists in large-scale disturbance scenarios between ecological integrity and human welfare (SER, 2008). The current ecological integrity of reclaimed lands in the oil sands mining region is estimated based on a limited set of data collected from reclamation areas up to 40 years old, while projections of future integrity are based on assumptions and empirical relationships derived from pyrogenic or forest-harvest disturbance regimes that may not apply perfectly or even well to reclaimed lands. Meanwhile, we account for human welfare in the reclaimed oil sands region primarily through the paradigm of predicted reclaimed end land uses. It appears that our approach is to demand near-continuous extractive capacity from our landscapes, no matter how severe the disturbances we impose upon them. However, transitioning directly from disturbance and extraction to expectations of renewed extractive capacity (land use) without identifying the functional requirements that are required to support this capacity may not represent a sound long-term strategy for land stewardship, particularly given the uncertainties outlined above with respect to current and projected future ecosystem function on reclaimed landscapes. The CEMA Reclamation Working Group's more recent work described above is in part focussed on identifying these functional requirements, and 
methods to assess their re-establishment. Based on evolving approaches within RWG, and an overall assessment of resource development and reclamation in the oil sands region, we suggest that we may need to incorporate a phase of recovery of ecosystem function, or "functional fallow" on reclaimed landscapes prior to considering renewed human uses of these landscapes, and that recognition of this phase (and corresponding delay of renewed use) should be incorporated into regulatory land management regimes.

\section{Acknowledgements}

The authors wish to particularly acknowledge Robert Vassov, in Environmental Research with Syncrude Canada Ltd., for his conceptual contributions to the framework and some of the approaches of this paper, and to the work programmes discussed above. In addition, we gratefully acknowledge the support and contributions of CEMA's Reclamation Working Group funders, participating members, and staff.

\section{References}

Alberta Environment (2010) Guidelines for Reclamation to Forest Vegetation in the Athabasca Oil Sands Region, 2nd edition, prepared by the Terrestrial Subgroup of the Reclamation Working Group of the Cumulative Environmental Management Association, Fort McMurray, AB., December 2009.

Alberta Environmental Protection (1994) The Alberta Regeneration Survey Manual (Addendum April 1997), Publication No.: Ref. 70.

(AR)115/1993 (1993) Government of Alberta. Environmental Protection and Enhancement Act Conservation and $\begin{array}{lllll}\text { Reclamation } & \text { Regulation, } & \text { (AR)115/1993, } & \text { viewed } & 7\end{array}$ http://www.qp.alberta.ca/documents/Regs/1993_115.pdf.

CEMA (2006) Land Capability Classification System for Forest Ecosystems in the Oil Sands, 3rd Edition, A document prepared for Alberta Environment by the Cumulative Environmental Management Association; Edmonton, AB.

Government of Alberta (2011) Oil sands website, Alberta Energy, viewed 7 May 2011, http://www.energy.gov.ab.ca/OilSands/oilsands.asp.

Oil Sands Vegetation Reclamation Committee (1998) Guidelines for Reclamation of Terrestrial Vegetation in the Oil Sands Region.

RSA (2000) C12. Province of Alberta, Environmental and Protection and Enhancement Act, viewed 7 May 2011, http://www.qp.alberta.ca/documents/Acts/E12.pdf.

RSA (2000) c.E-12, Approval No. 151469-00-01. Province of Alberta. Environmental Protection and Enhancement Act, Amending Approval.

SER (2008) Society for Ecological Restoration International Science \& Policy Working Group. The SER International Briefing Note Opportunities for Integrating Ecological Restoration \& Biological Conservation within the Ecosystem Approach, viewed 7 May 2011, www.ser.org \& Tucson: Society for Ecological Restoration International.

TECO Natural Resource Group Limited (2011a) Developing Baseline Site Index Estimates for the Ecosite Area Summary Table, Prepared for the Forest Productivity Task Group of the Reclamation Working Group of the Cumulative Environmental Management Association, Fort McMurray, Alberta, 32 p.

TECO Natural Resource Group Limited (2011b) Alberta Regeneration Standards for the Mineable Oil Sands Data Collection Field Protocols, Prepared for Alberta Sustainable Resource Development, Revised 21 March 2011.

Timberline Natural Resource Group Ltd. (2009) Assessment of Planting Densities in Reclaimed Landscapes Using the GYPSY Model, Technical Document, Prepared for the Cumulative Environmental Management Association, Reclamation Working Group, Terrestrial Subgroup, Fort McMurray, Alberta, p. 64. 